\title{
Comparison of Central Venous Catheter Related Deep Venous Thrombosis According to Insertion Site in an Intensive Care Unit of Bangladesh
}

\author{
Debasish Kumar Saha ${ }^{*}$, Suraiya Nazneen ${ }^{2}$, ASM Areef Ahsan ${ }^{3}$, Madhurima Saha4 ${ }^{4}$, Kaniz Fatema ${ }^{5}$, Fatema \\ Ahmed ${ }^{6}$, Rozina Sultana7
}

\begin{abstract}
Background: Central venous catheter (CVC) is usually inserted into subclavian, internal jugular, or femoral vein in critically ill patients. CVCs are associated with intravascular (infectious, thrombotic) and mechanical complications. CVC related deep venous thrombosis (DVT) is a common intravascular complication.
\end{abstract}

Objectives: To see the variation in occurrence of CVC related deep venous thrombosis according to different insertion site.

Method: It was a prospective observational study conducted in dept. of Critical Care Medicine, BIRDEM General Hospital; during a period of May, 2016 to July, 2019. Purposive sampling was conducted in patients fulfilling the selection criteria. The CVCs were percutaneously inserted using the Seldinger technique with standard operating procedure. After CVC insertion, patients were followed up daily to see any sign of deep venous thrombosis. After catheter removal [due to any cause including suspected catheter related blood stream infection (CRBSI), DVT, mechanical cause], all the study patients were investigated by duplex ultrasonography (USG) within 2 days for detection of venous thrombosis. The outcome was catheter related deep venous thrombosis (DVT).

Results: A total 349 patients, of which 167 (47.9\%) patients had CVC in subclavian, 88 (25.2\%) in internal jugular and 94 (26.9\%) in femoral vein. Total 12 patients were suspected to have catheter related DVT, but I I(3.2\%) patients were confirmed as DVT by duplex USG. DVT occurred significantly higher in femoral catheter site (8.5\%) than subclavian (I.8\%) and internal jugular site $(0 \%)$.

Conclusion: The occurrence of catheter related DVT was higher in femoral site than other two sites.

Key words: CVC, catheter related DVT, ICU

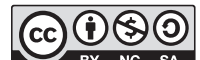

DOI: https://doi.org/I0.3329/jom.v23il.57932

Copyright: (C) 2022 Saha DK. This is an open access article published under the Creative Commons Attribution-NonCommercial-NoDerivatives 4.0 International License, which permits use, distribution and reproduction in any medium, provided the original work is properly cited, is not changed in any way and it is not used for commercial purposes.

Received: 05 September, 202I;

Accepted: 06 November, 2021

1. Assistant Professor, Dept. of Critical Care Medicine, BIRDEM, Dhaka

2. Registrar, Dept. of Critical Care Medicine, BIRDEM, Dhaka

3. Professor and Head, Dept. of Critical Care Medicine, BIRDEM, Dhaka

4. Registrar, Dept. of Critical Care Medicine, BIRDEM, Dhaka

5. Associate Professor, Dept. of Critical Care Medicine, BIRDEM, Dhaka

6. Associate Professor, Dept. of Critical Care Medicine, BIRDEM, Dhaka

7. Registrar, Dept. of Critical Care Medicine, BIRDEM, Dhaka *Corresponding author: Dr. Debasish Kumar Saha, Assistant Prof, Department of Critical Care Medicine, BIRDEM General Hospital, Dhaka. Cell: 01677952122 E-mail: debasish81dmc@yahoo.com

\section{Introduction}

Central venous catheter (CVC), also known as central line, or central venous access catheter, is a catheter placed into a large vein (subclavian, internal jugular, femoral vein). It is used to administer medications or fluids, obtain blood for tests and measure central venous pressure (in case of subclavian or internal jugular site). Central venous catheterizations are now common among critically ill patients.

Catheterization is associated with infectious, thrombotic, and mechanical complications. ${ }^{1}$ These complications can widely be catagorized into intravascular [includes catheter related blood stream infection (CRBSI), catheter tip 
colonization, catheter related deep vein thrombosis (DVT)] and mechanical (includes haemorrhage, haemothorax, pneumothorax, cardiac perforation, lost guidewire, catheter tip malposition etc).

The CVC related deep vein thrombosis (DVT) is an important intravascular complication which also has significant impact on morbidity and mortality. Thrombosis resulting from vascular catheter is of two types: fibrin sleeve (thrombin sheath) and vascular occlusive thrombosis (partial or total occlusion by mural thrombi). Most of the indwelling vascular catheters become engulfed in a thrombin sheath soon after insertion. ${ }^{2}$ Clinically, catheter related DVT is classified into symptomatic and asymptomatic. Although the importance of catheter related DVT has been debated, ${ }^{3}$ all thromboses have the potential to embolize. ${ }^{4}$

In numerous studies, the incidence of $\mathrm{CVC}$ related thrombosis has been evaluated. The percentage of clinically manifest thrombosis in these studies ranged from $0 \%$ to $12 \%{ }^{5}$ The role of the puncture-site of CVC insertion is still much debated in development of thrombosis. In a randomized trail (level 1) in ICU patients, insertion via the subclavian route had a low risk of major catheter related thrombosis as compared to a femoral route $(0 \%$ vs. $6 \%){ }^{6}$ Timsit et al. found the occurrence of catheter related thrombosis in a cohort (level 2) study in patients with subclavian vein CVCs as compared with jugular CVCs $(10.5 \%$ vs. $41.7 \%){ }^{7}$ In both studies patients were routinely screened by ultrasound for CVC related thrombosis. The occurrence of symptomatic deep vein thrombosis was found higher in femoral catheter site in comparison to internal jugular or subclavian sites. ${ }^{4}$ Additional factors reported to increase the risk of thrombosis are percutaneous insertion procedure, prior $\mathrm{CVC}$ at the same puncture site and a prolonged stay of the CVC for over 2 weeks. $^{5}$

The CVC related thrombosis have been reported to be associated with infective colonization of catheter tip. In a study in 265 critically ill patients, the risk of infection and sepsis was 2.6 fold increased in patients with catheter related thrombosis. ${ }^{7}$ In addition, CVC related infection may also increase the risk of subsequent clinically manifest thrombosis. In a study, CVC related infection increased the risk of thrombosis markedly in comparison with those without infection (24\% vs 3\%) (relative risk 17.6). ${ }^{5}$

The aim of this present study was to detect the insertion site wise occurrence of catheter related DVT

\section{Methods}

This prospective observational study was done during the period of May 2016 to July 2019 in Department of Critical Care Medicine, Bangladesh Institute of Research and Rehabilitation in Diabetes, Endocrine and Metabolic Disorders (BIRDEM) General Hospital, Dhaka. A total of 349 adult patients (age e" 18 years) requiring new CVC insertion for fluid management, medications, inotrope support and monitoring were enrolled in the study. Antibiotic coated CVC, tunnelled catheters, implantable devices, radiologically inserted catheters, dialysis catheters were excluded. CVC insertion was performed only after getting informed written consent from patient or his/her relative, when the platelet count was more than 50000/cumm and the international normalized ratio was less than 1.5 , as per ICU protocol. All catheterizations were performed either by an ICU consultant, registrar or a medical officer (with a minimum prior experience of at least $25 \mathrm{CVC}$ insertions under the supervision of a consultant). Insertion site selection was individualized by the treating physician. After CVC insertion, all the patients were followed up daily for any sign of catheter related DVT (pain, swelling, redness and tenderness of the affected limb). Removal of CVC was done when appropriate [development of catheter related blood steam infection (CRBSI), or DVT or no longer required]. All the information about the patient were collected by a structured data sheet and analysed by statistical package for the social sciences (SPSS) version 22.

\section{Result}

During the study period, $167 \mathrm{CVC}$ were inserted through subclavian vein, $88 \mathrm{CVC}$ through internal jugular vein and $94 \mathrm{CVC}$ through femoral vein. There was no significant difference among three groups in terms of age, gender distribution, presence of co-morbid illness (Table 1). Though most patients had more than one diagnosis during their admission, the primary cause/diagnosis was recorded. Table 2 showed the primary diagnosis of the study subjects at admission. The highest on admission diagnosis was pneumonia (22.6\%), followed by AKI (20.9\%), stroke (9.7\%) and acute pulmonary edema (4.9\%). Table 3 showed 12 patients were suspected to have DVT clinically, but 11 were confirmed by duplex USG; where the occurrence is significantly more in femoral than other two sites ( $p$ value $=0.002$ ). Table 4 showed the duration of CVC in venous site. 
Table 1 : Age, gender and co-morbidities of the study subjects $(n=349)$

\begin{tabular}{|c|c|c|c|c|c|}
\hline & \multicolumn{3}{|c|}{ Site } & \multirow{2}{*}{$\begin{array}{c}\text { Total } \\
(\mathrm{n}=349)\end{array}$} & \multirow[t]{2}{*}{$\mathrm{p}$ value } \\
\hline & Subclavia $n(n=167)$ & Internal jugular $(n=88)$ & Femoral $(n=94)$ & & \\
\hline Age (years) & $60.63 \pm 16.06$ & $63.00 \pm 14.38$ & $61.89 \pm 12.91$ & $61.57 \pm 14.84$ & $0.468^{\# \#}$ \\
\hline \multicolumn{6}{|l|}{ Gender } \\
\hline Male & $99(59.3)$ & $46(52.3)$ & $55(58.5)$ & $200(57.3)$ & $0.540^{\#}$ \\
\hline Female & $68(40.7)$ & $42(47.7)$ & $39(41.5)$ & $149(42.7)$ & \\
\hline \multicolumn{6}{|c|}{ Co-morbidities } \\
\hline $\mathrm{DM}$ & $121(96.8)$ & $65(97.0)$ & $80(97.6)$ & $266(97.1)$ & $0.950^{\#}$ \\
\hline HTN & $98(94.2)$ & $50(84.7)$ & $60(90.9)$ & $208(90.8)$ & $0.131^{\#}$ \\
\hline IHD & $16(40.0)$ & $12(40.0)$ & $8(26.7)$ & $36(36.0)$ & $0.444^{\#}$ \\
\hline Others & $13(40.6)$ & $13(50.0)$ & $14(46.7)$ & $40(45.5)$ & $0.765^{\#}$ \\
\hline
\end{tabular}

\#\#ANOVA test was done to measure the level of significance

${ }^{\#}$ Chi-square test was done to measure the level of significance

Table 2 : Primary diagnosis during ICU admission of the study subjects $(n=349)$

\begin{tabular}{lcc}
\hline Primary diagnosis & Frequency & Percentage \\
\hline Pneumonia & 79 & 22.6 \\
Acute Kidney Injury & 73 & 20.9 \\
Stroke & 34 & 9.7 \\
Acute Pulmonary Edema/LVF & 17 & 4.9 \\
Myocardial Infarction & 16 & 4.6 \\
Diabetic Ketoacidosis & 13 & 3.7 \\
Severe hyponatremia & 12 & 3.4 \\
Urosepsis & 11 & 3.2 \\
Meningitis & 9 & 2.6 \\
Hypoglycemia & 8 & 2.3 \\
Hyperglycemic Hyperosmolar State & 7 & 2.0 \\
Others & 79 & 22.6 \\
\hline Total & 349 & 100.0 \\
\hline
\end{tabular}

Table - 3: Catheter related DVT of the study subjects $(n=349)$

\begin{tabular}{lccccc}
\hline & \multicolumn{3}{c}{ Site } & Total & $\mathrm{p}$-value \\
\cline { 2 - 5 } & Subclavian(n=167) & Internal jugular(n=88) & Femoral(n=94) & $(\mathrm{n}=349)$ & \\
\hline Signs of catheter related thrombosis & $3(1.8)$ & $1(1.1)$ & $8(8.5)$ & $12(3.4)$ & 0.007 \\
Catheter related DVT & $3(1.8)$ & $0(0.0)$ & $8(8.5)$ & $11(3.2)$ & 0.002 \\
\hline
\end{tabular}

Chi-square test was done to measure the level of significance 
Table - 4: Duration of CV catheter and associated DVT of the study subjects $(n=349)$

\begin{tabular}{lccccc}
\hline & \multicolumn{3}{c}{ Site } & Total & p value \\
\cline { 2 - 4 } & Subclavian $(\mathrm{n}=167)$ & Internal jugular $(\mathrm{n}=88)$ & Femoral $(\mathrm{n}=94)$ & $(\mathrm{n}=349)$ & \\
\hline Duration of CV catheter & $7.3 \pm 5.1$ & $7.1 \pm 3.9$ & $6.4 \pm 3.1$ & $7.1 \pm 4.3$ & $0.158^{\# \#}$ \\
Duration of CVC & & & & \\
$\leq 10$ days & $134(80.2)$ & $67(76.1)$ & $80(85.1)$ & $281(80.5)$ & $0.309 \#$ \\
$>10$ days & $33(19.8)$ & $21(23.9)$ & $14(14.9)$ & $68(19.5)$ & \\
Catheter related DVT & & & & & \\
$\leq 10$ days & $2(1.5)$ & $0(0.0)$ & $8(10.0)$ & $10(3.6)$ & $0.001^{\#}$ \\
$>10$ days & $1(3.0)$ & $0(0.0)$ & $0(0.0)$ & $1(1.5)$ & $0.584^{\#}$ \\
\hline
\end{tabular}

\#\#ANOVA test was done to measure the level of significance

\#Chi-square test was done to measure the level of significance

\section{Discussion}

Complications associated with CVC have a major impact on the hospital course of patients admitted to ICU due to the morbidity, mortality and increased health care costs associated with them.

The overall incidence of catheter related DVT in this study was $3.2 \%$ which was within the range $(0 \%$ to $12 \%)$ as occurred in different studies (Van Rooden et al. 2005). In this study, the incidence of catheter related DVT in femoral site was $8.5 \%$, subclavian site was $1.8 \%$ and internal jugular site $0 \%$; and the occurrence is significantly greater in femoral site $(\mathrm{p}=0.002)$. This study produced similar findings as published by others. ${ }^{4,6}$ Merrer et al. found that femoral catheterization was associated with overall thrombotic complications in comparison to subclavian vein catheterization $(\mathrm{p}<0.001) .{ }^{6}$ Parienti et al. found that the occurrence of symptomatic DVT in subclavian catheters was $0.5 \%$, in internal jugular catheters was $0.9 \%$, in femoral catheters was $1.4 \% .{ }^{4}$ In this study, the higher occurrence of catheter related DVT in femoral site might be explained by reduced lower limb movement than other body parts causing venous stasis. Moreover, increased thrombogenicity due to microbial colonization might be a cause. ${ }^{8}$

Incidence of catheter related DVT varies with catheter composition, indication of catheterization (total parenteral nutrition, haemodialysis, cancer or ICU patient), fluids infused (chemotherapy, lipids or heparin), and diagnostic method for thrombosis. ${ }^{7}$ In this study, we tried to eliminate these factors. We used only non-tunnelled multi-lumen CVC for all patients. We didn't include haemodialysis catheter or CVC used for total parenteral nutrition, chemotherapy in this study. Routine $0.9 \%$ sodium chloride and heparin flush were administered in all $\mathrm{CV}$ catheterized patients according to our ICU protocol. We confirmed catheter related DVT by USG study, which was also used for venous thrombosis detection by other studies. $^{4,6,7}$ Prolonged duration of catheter ( $>10$ days) did not impact on the occurrence of DVT in different insertion sites (table 4) though prolonged stay of the CVC for over 2 weeks was a risk factor in some studies. ${ }^{5}$

\section{Conclusion}

CVC at femoral venous site was associated with more chance of development of DVT than subclavian or internal jugular venous site.

Conflict of interest: Nothing to declare.

\section{References}

1. McGee DC, Gould MK. Preventing complications of central venous catheterization. N Engl J Med 2003; 348:1123-1133.

2. Akmal AH, Hasan M, Marium A. The incidence of complications of central venous catheters at an intensive care unit. Annals of Thoracic Medicine 2007; 2(2): 61-63.

3. Parienti JJ, Mongardon N, Megarbane B, Mira JP, Kalfon P, Gros A, et al. Intravascular complications of central venous catheterization by insertion site. N Engl J Med 2015; 373:1220-1229.

4. Mansfield PF, Hohn DC, Fornage BD, Gregurch MA, Ota DM. Complications and failures of subclavian vein catheterization. N Engl J Med 1994; 331: 1735-1738.

5. Kaur R, Mathai AS, Abraham J. Mechanical and infectious complications of central venous catheterizations in a tertiary level intensive care unit in northern India. Indian Journal of Anaesthesia 2012; 56(4): 376-381.

6. Ruesch S, Walder B, Tramer MR. Complications of central venous catheters: Internal jugular versus subclavian access A systematic review. Critical Care Medicine 2002; 30(2): 454-460.

7. Gladwin MT, Slonim A, Landucci DL. Cannulation of the internal jugular vein: Is post procedural chest radiography always necessary? Crit Care Med 1999; 27:1819-1823.

8. Collter PE, Blocker SH, Graff DM. Cardiac tamponade from central venous catheters. Am J Surg.1998; 176: 212-214. 